\title{
EVALUACIÓN DE BISPIRIBAC-SODIO EN EL CONTROL DE MALEZAS EN ARROZ DE TEMPORAL ${ }^{1}$
}

\author{
Valentín Esqueda ${ }^{2}$ Enrique Rosales ${ }^{3}$
}

\begin{abstract}
RESUMEN
Evaluación de Bispiribac-sodio en el control de malezas en arroz de temporal. Se establecieron dos experimentos en Los Naranjos, Veracruz, México, con el objeto de evaluar la eficiencia biológica y el efecto fitotóxico del herbicida postemergente bispiribac-sodio, solo y en mezcla con otros herbicidas, para controlar malezas en el cultivo de arroz de temporal. Un experimento fue establecido el 13 de junio de 1999 el segundo el 9 de junio del 2000 con arroz de la variedad Milagro Filipino, a $100 \mathrm{~kg} / \mathrm{ha}$. El diseño experimental fue de bloques completos al azar con ocho tratamientos para el primero y seis para el segundo, y cuatro repeticiones de cada uno. En 1999, el control de malezas se evaluó a los 15, 30 y 105 días después de la aplicación (DDA) y en 2000 a los 15, 30 y 45 DDA. En ambos experimentos, la toxicidad se evaluó a los 15 y 30 DDA. Las especies de malezas dominantes fueron el zacate de agua [Echinochloa colona (L.) Link.], el pelillo ( $C y$ perus iria L.) y el zacate frente de toro [Digitaria ciliaris (Retz.) Koel]. Bispiribac-sodio afectó solamente plantas de malezas emergidas; este herbicida controló eficientemente al pelillo a $15 \mathrm{~g} / \mathrm{ha}$, al zacate de agua a $22 \mathrm{~g} / \mathrm{ha}$ y solamente tuvo un control parcial del zacate frente de toro. Al mezclar el bispiribac-sodio con clomazone o tiobencarbo, se incrementó el control de los zacates y se obtuvo un efecto herbicida residual. El bispiribac-sodio fue altamente selectivo al arroz.
\end{abstract}

\begin{abstract}
Evaluation of bispyribac-sodium for weed control in rainfed rice. Two experiments were established in Los Naranjos, Veracruz, México to evaluate the weed control efficacy of the postemergence herbicide bispyribac-sodium, alone and in mixture with other herbicides in rainfed rice and to evaluate the toxicity of bispyribac-sodium to rice. An experiment was established on June 13, 1999, and the second on June 9, 2000 with rice cv Milagro Filipino at $100 \mathrm{~kg} / \mathrm{ha}$. A randomized complete block design with eight treatments and the second six with four replication each one was established. In 1999, weed control was evaluated at 15, 30 and 105 days after application (DAA) and in 2000, at 15, 30 and 45 DAA. In both experiments, toxicity was evaluated at 15 and 30 DAA. The main weed species were junglerice [Echinochloa colona (L.) Link.], flatsedge (Cyperus iria L.) and Southern crabgrass [Digitaria ciliaris (Retz.) Koel]. Bispyribacsodium affected only emerged plants; this herbicide efficiently controlled flatsedge at $15 \mathrm{~g} / \mathrm{ha}$, junglerice at 22 $\mathrm{g} / \mathrm{ha}$ and Southern crabgrass was only partially controlled. When bispyribac-sodium was mixed with clomazone or thiobencarb, grass control was increased and a residual herbicide effect was obtained. Bispyribac-sodium was highly selective to rice.
\end{abstract}

\section{INTRODUCCIÓN}

El estado de Veracruz es el principal productor de arroz en la República Mexicana; donde se siembran anualmente entre 25.000 y 30.000 ha de las cuales apro- ximadamente el $80 \%$ se desarrolla en condiciones de temporal (Tosquy et al. 2002).

Uno de los factores que más afectan la producción de arroz de temporal, es la competencia de altas poblaciones

\footnotetext{
1 Recibido para publicación en abril del 2003. Presentado en la XLVIII Reunión Anual del PCCMCA, Santo Domingo, República Dominicana. 2002.

2 Campo Experimental Cotaxtla. CIRGOC. INIFAP. SAGARPA. vesqueda@ prodigy.net.mex.

3 Campo Experimental Río Bravo. CIRNE. INIFAP. SAGARPA.
} 
de malezas, principalmente zacates anuales, como el zacate de agua [Echinochloa colona (L.) Link.] y ciperáceas, que en Veracruz, han llegado a cuantificarse en poblaciones superiores a los 20 millones de plantas/ha (Esqueda y Acosta 1985). Si las malezas no son controladas, o bien si su control es deficiente, interfieren con el desarrollo del arroz, pudiendo reducir su producción de grano entre 85 y 100\% (Esqueda 1990).

Para controlar las malezas, los agricultores utilizan comunmente al herbicida propanil aplicado en postemergencia, solo o en mezcla con 2,4-D. Debido a que el propanil no tiene residualidad, para minimizar el daño de la maleza al arroz es necesario aplicarlo al menos en dos ocasiones durante el período crítico de competencia (Esqueda 1998). Una de las limitantes del propanil, es que solamente es eficiente para controlar zacates anuales pequeños y muestra controles irregulares o deficientes con zacates perennes o zacates anuales de tamaño grande (Esqueda 1986, Leah et al. 1995). En la actualidad, se está incrementando el uso del propanil en mezcla con herbicidas residuales como el clomazone y la pendimetalina, que aplicados en una sola ocasión en postemergencia temprana, controlan la maleza hasta el "cierre" del cultivo (Crawford y Jordan 1995, Esqueda 2000).

El propanil ha sido utilizado por más de 40 años (Smith, 1961) y su aplicación contínua ha ocasionado que en varios países hayan aparecido biotipos de varias especies de Echinochloa con resistencia a este herbicida (Giannopolitis y Vassiliou 1989; Fischer et al. 1993, Riches et al. 1997). Recientemente, en México se detectaron biotipos de zacate de agua con tolerancia al propanil, en arrozales de los estados de Veracruz y Campeche (Bolaños et al. 2001). Lo anterior señala la necesidad, de que este herbicida debe paulatinamente reemplazarse por otros productos con diferente modo de acción.

Bispiribac-sodio, es un herbicida postemergente no residual, que inhibe la enzima acetolactato sintasa, la cual es esencial para biosintetizar los aminoácidos valina, leucina e isoleucina (Retzinger y Mallory-Smith, 1997). A diferencia del propanil, cuya acción es relativamente rápida, el bispiribac-sodio requiere entre dos y tres semanas para eliminar las malezas y puede incluso controlar zacates que se encuentran en la etapa de amacollamiento, lo que difícilmente se logra con el propanil (Valent 2000). Como bispiribac-sodio tiene un modo de acción diferente al del propanil, puede ser útil para prevenir o retrasar la aparición de biotipos de malezas con resistencia a este herbicida, o para controlar aquellos que ya han desarrollado ésta. En México, bispiribac-sodio no se ha evaluado con anterioridad, por lo que se condujo una investigación para determinar si es- te herbicida, puede ser incorporado como una nueva opción para combatir malezas en el cultivo de arroz en nuestro país.

\section{Objetivos}

Evaluar la eficiencia biológica del bispiribac-sodio solo y en mezcla con otros herbicidas para controlar malezas en el cultivo de arroz de temporal.

Evaluar el efecto fitotóxico del bispiribac-sodio en el arroz.

\section{MATERIALES Y MÉTODOS}

Se condujeron dos experimentos en un terreno localizado en el Ejido Los Naranjos, Mpio. de Tres Valles, Veracruz. Uno de los experimentos fue establecido en el ciclo de temporal de 1999 (experimento 1) y el otro en el de 2000 (experimento 2).

Experimento 1. La siembra del lote experimental se efectuó el 13 de junio de 1999. Se utilizó semilla de arroz de la variedad Milagro Filipino, sembrada en surcos a una densidad de $100 \mathrm{~kg} / \mathrm{ha}$. El arroz se fertilizó a los 36 y 62 días después de la emergencia (DDE). En ambas ocasiones se usó la cantidad de urea equivalente a $65 \mathrm{~kg}$ de N/ha, aplicada al voleo sobre suelo húmedo.

Se evaluaron ocho tratamientos incluyendo un testigo sin aplicar (Cuadro 1).

Los tratamientos estuvieron distribuídos en el terreno de acuerdo a un diseño experimental de bloques completos al azar con cuatro repeticiones. Las parcelas experimentales constaron de diez surcos de $10 \mathrm{~m}$ de longitud y $0,30 \mathrm{~m}$ de separación entre surcos.

Los tratamientos 4 y 5 se aplicaron cuando el arroz había desarrollado entre tres y cuatro hojas y tenía una altura de 14 a $17 \mathrm{~cm}$. Los zacates dominantes tenían entre dos y cinco hojas y una altura entre 2 y $8 \mathrm{~cm}$. Los tratamientos 1, 2, 3 y 6 se aplicaron a los 13 DDE, cuando el arroz tenía entre cuatro y cinco hojas y una altura de 18 a $22 \mathrm{~cm}$; los zacates dominantes tenían entre tres y seis hojas y una altura de 10 a $20 \mathrm{~cm}$. El tratamiento 7 consistió en dos aplicaciones: la primera con la mezcla de propanil + 2,4-D, efectuada a los 8 DDE y la segunda, con propanil a los 22 DDE.

Experimento 2. La siembra del experimento se efectuó el 9 de junio de 2000. Se utilizó la misma variedad, densidad de siembra y fertilización indicada para 
Cuadro 1. Descripción de tratamientos en 1999. Los Naranjos, mpio. de Tres Valles, Veracruz. 1999.

\begin{tabular}{llcl}
\hline No. & \multicolumn{1}{c}{ Tratamiento* $^{*}$} & $\begin{array}{c}\text { Dosis } \\
\text { (g i.a./ha) }\end{array}$ & $\begin{array}{c}\text { Epoca de } \\
\text { aplicación }\end{array}$ \\
\hline 1 & Bispiribac-sodio & 15 & $13 \mathrm{DDE}$ \\
2 & Bispiribac-sodio & 22 & $13 \mathrm{DDE}$ \\
3 & Bispiribac-sodio & 30 & $13 \mathrm{DDE}$ \\
4 & Bispiribac-sodio + tiobencarbo & $22+3500$ & $8 \mathrm{DDE}$ \\
5 & Bispiribac-sodio + clomazone & $22+480$ & $8 \mathrm{DDE}$ \\
6 & Bispiribac-sodio + 2,4-D & $22+479$ & $13 \mathrm{DDE}$ \\
7 & Propanil + 2,4-D/propanil** & $2880+240 /$ & $8 \mathrm{DDE} /$ \\
& & 2880 & $22 \mathrm{DDE}$ \\
8 & Testigo sin aplicar & & \\
\hline
\end{tabular}

* A los tratamientos del 1 al 5 se les agregó el coadyuvante y humectante Kinetic en dosis de $125 \mathrm{~mL}$ por cada $100 \mathrm{~L}$ de agua ** Testigo del agricultor.

el experimento 1. Se evaluaron seis tratamientos, los cuales se describen el el Cuadro 2.

El diseño experimental y las dimensiones de las parcelas experimentales fueron los mismos que se indican para el experimento 1. Los tratamientos 1,2 y 3 y la primer parte de los tratamientos 4 y 5 se aplicaron cuando el arroz había desarrollado de cinco a seis hojas y un macollo y tenía una altura entre 18 a $23 \mathrm{~cm}$. Los zacates dominantes tenían de dos a seis hojas y una altura entre 8 y $20 \mathrm{~cm}$. La segunda parte de los tratamientos cuatro y cinco se aplicó cuando el arroz tenía de ocho a nueve hojas, dos macollos y una altura de 28 a $35 \mathrm{~cm}$; a su vez, los zacates dominantes que no habían emergido al momento de la primera aplicación, o que no

Cuadro 2. Descripción de tratamientos en 2000. Los Naranjos, mpio. de Tres Valles, Veracruz. 1999.

\begin{tabular}{llcc}
\hline No. & \multicolumn{1}{c}{ Tratamiento* } & $\begin{array}{c}\text { Dosis } \\
\text { (g i.a./ha) }\end{array}$ & $\begin{array}{c}\text { Epoca de } \\
\text { aplicación }\end{array}$ \\
\hline 1 & Bispiribac-sodio & 15 & $15 \mathrm{DDE}$ \\
2 & Bispiribac-sodio & 22 & $15 \mathrm{DDE}$ \\
3 & Bispiribac-sodio & 30 & $15 \mathrm{DDE}$ \\
4 & Bispiribac-sodio / bispiribac- & $22+22$ & $15 \mathrm{DDE} /$ \\
& sodio & & $30 \mathrm{DDE}$ \\
5 & Propanil + 2,4-D/propanil** & $2880+240 /$ & $15 \mathrm{DDE} /$ \\
& & 2880 & $30 \mathrm{DDE}$ \\
6 & Testigo sin aplicar & & \\
\hline
\end{tabular}

* A los tratamientos del 1 al 5 se les agregó el coadyuvante y humectante Kinetic en dosis de $125 \mathrm{~mL}$ por cada $100 \mathrm{~L}$ de agua.

** Testigo del agricultor. fueron controlados por ésta, tenían de tres a cinco macollos y una altura de 5 a $35 \mathrm{~cm}$ (como se aprecia en el Cuadro 2, los tratamientos 4 y 5 fueron aplicaciones secuenciales, la primera parte del tratamiento se aplicó a los 15 DDE y la segunda a los $30 \mathrm{DDE}$, por lo tanto, la segunda parte se refiere a la segunda aplicación de que constó cada uno de los tratamientos antes indicados).

En 1999, la densidad de población de malezas se determinó a los 8, 13 y 118 DDE, coincidiendo con la época de la aplicación de los tratamientos y de la madurez del cultivo, respectivamente. A su vez, en 2000, lo anterior se llevó a cabo a los 15 y 30 DDE. Los conteos se realizaron con un cuadrante de $0,5 \times 0,5 \mathrm{~m}$, el cual fue lanzado al azar, en cada una de las parcelas correspondientes a los testigos sin aplicar.

El efecto de los tratamientos herbicidas se evaluó visualmente en las especies de malezas dominantes a los 15, 30 y 105 días después de la aplicación (DDA) en el experimento 1 y a los 15,30 y 45 DDA en el experimento 2. Se utilizó la escala de 0 a $100 \%$, en donde 0 significó que la maleza no fue afectada y $100 \%$ que fue completamente destruída. La evaluación visual del control de malezas mediante la escala porcentual es ampliamente utilizada, ejemplos de ello en arroz se tienen en Ntanos et al. 2000 y Esqueda 2000.

Se evaluó visualmente la toxicidad de los herbicidas al arroz a los 15 y 30 DDA. Se asignaron valores en la escala de 0 a $100 \%$, en donde 0 significó que el arroz no fue afectado y $100 \%$ que fue completamente destruído.

Para homogenizar las varianzas, los datos experimentales de control de malezas fueron transformados a su valor de arco seno $\%$, de acuerdo a lo que recomiendan Gomez y Gomez (1984). Los análisis de varianza se efectuaron con los datos transformados y como prueba de separación de medias se utilizó Tukey $(0,05)$. Por motivos de claridad, los porcentajes de control de malezas se presentan con los datos originales.

\section{RESULTADOS Y DISCUSIÓN}

Experimento 1. La población total de malezas fue de 8'990,000 plantas/ha a los 8 DDE y aumentó hasta 11 '260,000 plantas/ha a los 13 DDE, para finalizar en 775,000 plantas/ha a los 118 DDE. Las principales especies de malezas presentes en el sitio experimental fueron: el zacate de agua [E. colona (L.) Link.], el zacate frente de toro [Digitaria ciliaris (Retz.) Koel] y el pelillo [Cyperus iria L.]. E. colona fue la especie dominante en las etapas iniciales del cultivo, ocupando entre el 93,8 y $95,3 \%$ de la población total de malezas; sin 
Cuadro 3. Densidad de población de malezas (plantas/ha) a los 8, 13 y 118 DDE en 1999. Los Naranjos, mpio. de Tres Valles, Ver. 1999.

\begin{tabular}{lrrr}
\hline \multicolumn{1}{c}{ Especie } & $\mathbf{8}$ DDE & 13 DDE & 118 DDE \\
\hline Echinochloa colona & 8.430 .000 & 10.730 .000 & 240.000 \\
Cyperus iria & 260.000 & 150.000 & 0 \\
Digitaria ciliaris & 170.000 & 180.000 & 410.000 \\
Otras & 130.000 & 200,000 & 125.000 \\
\hline Total & $\mathbf{8 . 9 9 0 . 0 0 0}$ & $\mathbf{1 1 . 2 6 0 . 0 0 0}$ & $\mathbf{7 7 5 . 0 0 0}$ \\
\hline
\end{tabular}

embargo, a los 118 DDE, las mayoría de las plantas de esta especie habían muerto y su lugar había sido ocupado por plantas de D. ciliaris, que se convirtió en la especie dominante (Cuadro 3).

A los 15 DDA, se tuvo un control total de E. colo$n a$, con la aplicación de bispiribac-sodio + clomazone y casi total con bispiribac-sodio + tiobencarbo; a su vez, la mezcla de propanil $+2,4-\mathrm{D}$, complementada con una segunda aplicación de propanil y el bispiribac-sodio aplicado en su dosis más alta proporcionaron controles de esta especie de $90 \%$, siendo estadísticamente semejantes a los de los tratamientos en que el bispiribac-sodio se mezcló con clomazone o con tiobencarbo. En esta etapa, las dosis media y baja de bispiribac-sodio mostraron controles de E. colona de entre 80 y $85 \%$, mientras que la mezcla de bispiribac-sodio + 2,4-D tenía un control de $76 \%$, siendo el tratamiento químico con el control más bajo de esta especie. A los 30 DDA, las mezclas de bispiribac-sodio + clomazone o bispiribacsodio + tiobencarbo, así como el bispiribac-sodio a 30 $\mathrm{g} / \mathrm{ha}$, mostraron un control total de E. colona, mientras que se observó un incremento significativo en el efecto sobre esta especie por parte de bispiribac-sodio en sus dosis media y baja y con la mezcla de bispiribac-sodio $+2,4-\mathrm{D}$. El control obtenido con la mezcla de propanil $+2,4-\mathrm{D}$ seguido de propanil, se mantuvo sin cambios respecto al control observado en la evaluación anterior. A los 105 DDA, bispiribac-sodio + clomazone, mantenía un control total de E. colona, mientras que bispiribac-sodio + tiobencarbo, propanil + 2,4-D seguido de propanil y bispiribac-sodio a $30 \mathrm{~g} / \mathrm{ha}$, tenían controles de entre 95 y $97 \%$. Por su parte, los controles de bispiribac-sodio a 15 y $22 \mathrm{~g} /$ ha y la mezcla de bispiribac-sodio + 2,4-D oscilaron entre el 83 y $89 \%$ (Cuadro 4).

El efecto de los tratamientos sobre D. ciliaris solamente fue posible evaluarlo a los 105 DDA. En las parcelas de los testigos sin aplicar, se observó que un alto porcentaje de la población de esta especie emergió después de los $13 \mathrm{DDE}$, lo cual tuvo como consecuencia, que solamente con el tratamiento con doble aplicación de propanil y con bispiribac-sodio mezclado con clo- mazone y con tiobencarbo (que son herbicidas residuales), se tuvieran controles muy buenos o buenos de esta especie. Como el bispiribac-sodio no tiene efecto residual, al aplicarlo individualmente, solo proporcionó controles de D. ciliaris de entre 35 y $55 \%$, siendo el control directamente proporcional a la dosis aplicada. Cuando el bispiribac-sodio a $22 \mathrm{~g} / \mathrm{h}$ a se mezcló con 2,4$\mathrm{D}$, el control de $D$. ciliaris, fue ligeramente menor al obtenido con la misma dosis de bispiribac-sodio solo, lo cual puede ser indicación de un posible antagonismo entre estos dos herbicidas como se ha observado cuando se mezcla el fenoxaprop-etil con el 2,4-D (Esqueda 1998b) (Cuadro 4). (Como puede observarse en el Cuadro 3, la densidad de población de $D$. ciliaris era bajísima en comparación de lade E. Colona, lo que ocasionó que no hubiera suficientes plantas para asignarles un valor de control). Cuando E. Colona maduró y murió por causas naturales, entonces $D$. ciliris que se había mantenido restringida u oculta pudo expresarse tanto en los testigos laterales, como en las parcelas tratadas, por lo anterior, se realizó la evaluación a los 105 DDA).

En ninguna de las dos épocas de evaluación de toxicidad se observaron daños ocasionados por los tratamientos herbicidas a las plantas de arroz.

Experimento 2. En el sitio experimental, se tuvo una población de malezas de 3.275.000 plantas/ha a los 15 DDE y se redujo a 1.385 .000 a los 30 DDE. Las especies dominantes fueron C. iria, E. colona y D. ciliaris, que en conjunto ocuparon el 89,9 y $78,3 \%$ de la población total de malezas, en la primera y segunda época de conteos, respectivamente (Cuadro 5).

Cuadro 4. Porcenaje de control de los tratamientos sobre E. colona a los 15, 30 y 105 DDA y D. ciliaris a los 105 DDA. Los Naranjos, mpio. de Tres Valles, Ver. 1999.

\begin{tabular}{llllll}
\hline \multicolumn{1}{c}{ Tratamiento } & \multicolumn{4}{c}{ E. colona } & D. ciliaris \\
\cline { 2 - 6 } & 15 DDA 30 DDA & 105 DDA & 105 DDA \\
\hline Bis $(15 \mathrm{~g})$ & $83 \mathrm{bc} *$ & $95 \mathrm{~b}$ & $83 \mathrm{~d}$ & $35 \mathrm{c}$ \\
Bis $(22 \mathrm{~g})$ & $85 \mathrm{bc}$ & $98 \mathrm{ab}$ & $89 \mathrm{~cd}$ & $49 \mathrm{bc}$ \\
Bis $(30 \mathrm{~g})$ & $90 \mathrm{ab}$ & $100 \mathrm{a}$ & $95 \mathrm{bc}$ & $55 \mathrm{bc}$ \\
Bis + Tio $(22 \mathrm{~g}+3500 \mathrm{~g})$ & $99 \mathrm{a}$ & $100 \mathrm{a}$ & $97 \mathrm{~b}$ & $82 \mathrm{ab}$ \\
Bis + Clo $(22 \mathrm{~g}+480 \mathrm{~g})$ & $100 \mathrm{a}$ & $100 \mathrm{a}$ & $100 \mathrm{a}$ & $97 \mathrm{a}$ \\
Bis + 2,4-D $(22 \mathrm{~g}+479 \mathrm{~g})$ & $76 \mathrm{c}$ & $99 \mathrm{ab}$ & $85 \mathrm{~d}$ & $38 \mathrm{c}$ \\
Pro + 2,4-D/Pro $(2880+$ & & & & \\
240/2880 g) & $90 \mathrm{ab}$ & $90 \mathrm{~b}$ & $96 \mathrm{bc}$ & $98 \mathrm{a}$ \\
Testigo sin aplicar & $0 \mathrm{~d}$ & $0 \mathrm{c}$ & $0 \mathrm{e}$ & 0 & $\mathrm{~d}$ \\
\hline
\end{tabular}

Bis $=$ Bispiribac-sodio, Tio $=$ Tiobencarbo, Clo $=$ Clomazone , Pro $=$ Propanil.

*Tukey 0,05 . 
Cuadro 5. Densidad de población de malezas (plantas/ha) a los 15 y 30 DDE en 2000. Los Naranjos, mpio. de Tres Valles, Ver. 1999.

\begin{tabular}{lrr}
\hline \multicolumn{1}{c}{ Especie } & 15 DDE & 30 DDE \\
\hline Cyperus iria & 1.690 .00 & 575.000 \\
Echinochloa colona & 970.000 & 310.000 \\
Digitaria ciliaris & 285.000 & 200.000 \\
Otras & 330.000 & 300.000 \\
Total & $\mathbf{3 . 2 7 5 . 0 0 0}$ & $\mathbf{1 . 3 8 5 . 0 0 0}$ \\
\hline
\end{tabular}

A los 15 DDA, todos los tratamientos herbicidas tuvieron un control de $C$. iria superior al $90 \%$, destacando la mezcla propanil + 2,4-D seguida de propanil con $99 \%$ y la dosis mayor de bispiribac-sodio con $98 \%$. En los tratamientos en que se aplicó el bispiribac-sodio a $22 \mathrm{~g} / \mathrm{ha}$ y en doble aplicación, el control fue de $96 \%$, siendo estos valores estadísticamente semejantes a los de los tratamientos antes mencionados. A su vez, bispiribac-sodio a $15 \mathrm{~g} / \mathrm{ha}$ ofreció un control de $92 \%$, el cual fue semejante al de todos los otros tratamientos en que se aplicó este herbicida, pero inferior al de propanil + 2,4-D seguido por propanil. A los 30 DDA, se observó un control total de $C$. iria con la mezcla de propanil + 2,4-D seguida de propanil y con el tratamiento de doble aplicación de bispiribac-sodio, mientras que en los tres tratamientos de bispiribac-sodio, el control osciló entre 93 y $98 \%$, sin haber diferencia estadística signifcativa entre ellos. En la última evaluación, se tuvieron controles de C. iria de entre 97 y 100\%, lo cual es una indicación de que esta especie es relativamente fácil de controlar por cualquiera de los tratamientos evaluados (Cuadro 6).

Cuadro 6. Porcenaje de control de los tratamientos sobre C. iria a los 15, 30 y 45 DDA. Los Naranjos, mpio. de Tres Valles, Veracruz. 1999.

\begin{tabular}{llll}
\hline \multicolumn{1}{c}{ Tratamiento } & 15 DDA & 30 DDA & 45 DDA \\
\hline Bis (15 g) & $92 \mathrm{~b}^{*}$ & $93 \mathrm{~b}$ & $98 \mathrm{~b}$ \\
Bis (22 g) & $96 \mathrm{ab}$ & $97 \mathrm{~b}$ & $97 \mathrm{~b}$ \\
Bis (30 g) & $98 \mathrm{ab}$ & $98 \mathrm{~b}$ & $98 \mathrm{~b}$ \\
Bis / Bis (22 / 22 g) & $96 \mathrm{ab}$ & $100 \mathrm{a}$ & $100 \mathrm{a}$ \\
Pro + 2,4-D/Pro (2880+ & & & \\
240/2880 g) & $99 \mathrm{a}$ & $100 \mathrm{a}$ & $99 \mathrm{ab}$ \\
Testigo sin aplicar & $0 \mathrm{c}$ & $0 \mathrm{c}$ & $0 \mathrm{c}$ \\
\hline
\end{tabular}

Bis $=$ Bispiribac-sodio $\quad$ Pro $=$ Propanil.

*Tukey 0,05 .
Con el tratamiento de doble aplicación de bispiribac-sodio, se tuvo un control total de E. colona, desde los 15 DDA hasta los 30 DDA, observándose a los 45 DDA una ligera reinfestación de plantas que emergieron después de la segunda aplicación. Las dosis de 22 y $30 \mathrm{~g} / \mathrm{ha}$ de bispiribac-sodio, se comportaron en forma semejante durante las tres épocas de evaluación, llegando a ofrecer un $100 \%$ de control a los 30 DDA y disminuyendo ligeramente su efecto a los 45 DDA. Cuando fue aplicado a la dosis de $15 \mathrm{~g} / \mathrm{ha}$, el bispiribac-sodio, también fue eficiente para controlar E. colona, sobre todo a los 30 y 45 DDA. La mezcla de propanil + 2,4-D seguida de propanil tuvo problemas para controlar $E$. colona, ofreciendo un $69 \%$ de control en la evaluación inicial, para terminar en $48 \%$ a los 45 DDA (Cuadro 7). Lo anterior, refleja la superioridad del bispiribac-sodio sobre el propanil, para controlar zacates de gran tamaño o que han amacollado.

Cuadro 7. Porcenaje de control de los tratamientos sobre E. colona a los 15, 30 y 45 DDA. Los Naranjos, mpio. de Tres Valles, Veracruz. 1999.

\begin{tabular}{llll}
\hline \multicolumn{1}{c}{ Tratamiento } & 15 DDA & 30 DDA & 45 DDA \\
\hline Bis $(15 \mathrm{~g})$ & $91 \mathrm{~b}$ & $99 \mathrm{a}$ & $95 \mathrm{~b}$ \\
Bis $(22 \mathrm{~g})$ & $96 \mathrm{~b}$ & $100 \mathrm{a}$ & $98 \mathrm{ab}$ \\
Bis $(30 \mathrm{~g})$ & $97 \mathrm{~b}$ & $100 \mathrm{a}$ & $98 \mathrm{ab}$ \\
Bis / Bis (22/22 g) & $100 \mathrm{a}$ & $100 \mathrm{a}$ & $98 \mathrm{a}$ \\
Pro + 2,4-D/Pro (2880+ & & & \\
240/2880 g) & $69 \mathrm{c}$ & $55 \mathrm{~b}$ & $48 \mathrm{c}$ \\
Testigo sin aplicar & $0 \mathrm{~d}$ & $0 \mathrm{c}$ & $0 \mathrm{~d}$ \\
\hline
\end{tabular}

Bis $=$ Bispiribac-sodio, Pro $=$ Propanil.

*Tukey 0,05 .

Debido a que en las etapas iniciales de desarrollo las plantas de $D$. ciliaris tienen un hábito de crecimiento semipostrado y a su relativamente baja densidad de población, en un inicio fueron parcial o totalmente cubiertas por el follaje de las plantas de E. colona, cuyas poblaciones fueron mucho mayores. Lo anterior disminuyó el contacto del herbicida bispiribac-sodio con su follaje y se manifiestó en controles muy deficientes y paulatinamente nulos de esta especie. El efecto que tiene un buen contacto del herbicida bispiribac-sodio en el control de $D$. ciliaris se manifestó con la doble aplicación de este herbicida, ya que con la primera aplicación, se eliminaron las plantas de E. colona, por lo que la segunda aplicación pudo tener un mejor contacto con el follaje de $D$. ciliaris. Aun así, el control final de esta especie con bispiribac-sodio, fue de $75 \%$, mientras que 
con la mezcla de propanil + 2,4-D seguida de propanil, en todas las evaluaciones su control fue superior al $90 \%$, indicando una mayor susceptibilidad de D. ciliaris al propanil que al bispiribac-sodio (Cuadro 8).

Cuadro 8. Porcenaje de control de los tratamientos sobre $D$. $c i$ liaris a los 15, 30 y 45 DDA. Los Naranjos, mpio. de Tres Valles, Ver. 1999.

\begin{tabular}{llll}
\hline \multicolumn{1}{c}{ Tratamiento } & 15 DDA & 30 DDA & 45 DDA \\
\hline Bis $(15 \mathrm{~g})$ & $25 \mathrm{c}$ & $0 \mathrm{~d}$ & $0 \mathrm{c}$ \\
Bis $(22 \mathrm{~g})$ & $33 \mathrm{bc}$ & $0 \mathrm{~d}$ & $0 \mathrm{c}$ \\
Bis $(30 \mathrm{~g})$ & $35 \mathrm{~b}$ & $18 \mathrm{c}$ & $0 \mathrm{c}$ \\
Bis / Bis $(22 / 22 \mathrm{~g})$ & $38 \mathrm{~b}$ & $60 \mathrm{~b}$ & $75 \mathrm{~b}$ \\
Pro + 2,4-D/Pro $(2880+$ & & & $92 \mathrm{a}$ \\
240/2880 g) & $95 \mathrm{a}$ & $100 \mathrm{a}$ & $0 \mathrm{c}$ \\
Testigo sin aplicar & $0 \mathrm{~d}$ & $0 \mathrm{c}$ & 0 \\
\hline
\end{tabular}

Bis $=$ Bispiribac-sodio, Pro $=$ Propanil.

*Tukey 0,05 .

Al igual que en el experimento conducido en 1999, no se observaron síntomas de toxicidad al arroz con ninguno de los tratamientos aplicados.

Es importante recalcar, que aunque el herbicida bispiribac-sodio aplicado individualmente mostró un buen control del primer flujo de emergencia de E. colo$n a$, no puede ser recomendado como un tratamiento único para el control de las malezas del arroz de temporal. Lo anterior, se debe a que una vez que es eliminado el primer flujo de emergencia de malezas, al no ser el bispiribac-sodio un herbicida residual, se presentan nuevos flujos de emergencia de la misma u otras especies de malezas, como en el caso de este experimento, en que E. colona fue desplazada por $D$. ciliaris después de la aplicación del bispiribac-sodio. Así pues, para obtener un control eficiente de las malezas durante el período de tiempo requerido por el arroz, el bispiribacsodio debería aplicarse en dos ocasiones, complementarse con una segunda aplicación de propanil o aplicarse en mezcla con clomazone o tiobencarbo.

\section{CONCLUSIONES}

El primer flujo de emergencia de E. colona fue controlado eficientemente con bispiribac-sodio a partir de $22 \mathrm{~g} / \mathrm{ha}$.

Una sola aplicación de bispiribac-sodio + clomazone o de bispiribac-sodio + tiobencarbo, fue suficiente para controlar E. colona durante todo el ciclo del arroz.
Bispiribac-sodio puede utilizarse para el control de C. iria desde $15 \mathrm{~g} / \mathrm{ha}$.

Bispiribac-sodio solo tuvo un control parcial de $D$. ciliaris, el cual se incrementa considerablemente cuando se le añadió clomazone o tiobencarbo.

La mezcla de propanil + 2,4-D seguida de propanil tuvo un mejor efecto sobre $D$. ciliaris que el bispiribacsodio sólo o en aplicación doble.

El bispiribac-sodio no ocasionó toxicidad al arroz y puede utilizarse con seguridad en este cultivo.

\section{LITERATURA CITADA}

BOLAÑOS, E. A.; VILLA; J. T.; VALVERDE, B. E. 2001. Respuesta de Echinochloa colona (L.) Link a propanil en áreas arroceras selectas de México. Revista Mexicana de la Ciencia de la Maleza 1(2):21-26.

CRAWFORD, S. H.; JORDAN, D. L. 1995. Comparison of single and multiple applications of propanil and residual herbicides in dry-seeded rice (Oryza sativa). Weed Technology 9:153-157.

ESQUEDA, V. A. 1986. Efecto de la dosis y época de aplicación de propanil + 2,4-D amina en el control de la maleza y rendimiento del arroz de temporal. In: SOMECIMA (ed.). Memorias del VII Congreso Nacional de la Ciencia de la Maleza. Guadalajara, Jal., México. p. 391-397.

ESQUEDA E., V. A. 1990. La maleza y su control en arroz de temporal en México. Series Técnicas de ASOMECIMA 1(1):12-16.

ESQUEDA, V. A. 1998a. Comportamiento de la mezcla de clomazone + propanil + 2,4-D en el control de malezas en arroz (Oryza sativa L.) en postemergencia temprana. In: F. Guevara Fefer (ed.) Memorias del XIX Congreso Nacional del Congreso de la Maleza. Mexicali, B. C., México. p. 86-92.

Esqueda E., V. A. 1998b. Efecto de dosis y épocas de aplicación del herbicida fenoxaprop-etil en arroz (Oryza sati$v a$ L.) de temporal. In: F. Guevara Fefer (ed.). Memorias del XIX Congreso Nacional del Congreso de la Maleza. Mexicali, B. C., México. p. 81-85.

ESQUEDA, V. A. 2000. Control de malezas en arroz de temporal con clomazone, solo y en mezcla con propanil y 2,4-D. Agronomía Mesoamericana 11(1):51-56.

ESQUEDA E., V. A.; ACOSTA, S. 1985. Daños y control de las malas hierbas en el cultivo del arroz de temporal en el centro del estado de Veracruz y norte de Oaxaca. SARH. INIA. Folleto de Investigación No. 65. 60 p. 
FISCHER, A. J., GRANADOS, E.; TRUJILLO, D. 1993. Propanil resistance in populations of junglerice (Echinochloa colona) in Colombia rice fields. Weed Science 41:201-206

GIANNOPOLITIS, C. N.; VASSILIOU, G. 1989. Propanil tolerance in Echinochloa crus-galli (L.) Beauv. Tropical Pest Management 35:6-7.

GÓMEZ, K. A.; GÓMEZ, A. 1984. Statistical Procedures for Agricultural Research. 2nd. ed. New York, J. Wiley \& Sons. $680 \mathrm{p}$.

LEAH, J. M.; CASELEY, J. C.; RICHES, C. R.; VALVERDE, B. E. 1995. Age-related mechanisms of propanil tolerance in jungle-rice, Echinochloa colona. Pesticide Science 43:347-354.

NTANOS, D. A., KOUTROUBAS, S. D.; MAVROTAS, C. 2000. Barnyardgrass (Echinochloa crus-galli) control in water-seeded rice (Oryza sativa) with cyhalofopbutyl. Weed Technology 14(2): 383-388.
RETZINGER, E. J.; MALLORY, C. 1997. Classification of herbicides by site of action for weed resistance management strategies. Weed Technology 11:384-393.

RICHES, C. R.; KNIGHTS, S.; CHAVES, L.; CASELEY, J. C.; VALVERDE, B. E. 1997. The role of pendime thalin in the integrated management of propanil-resistant Echinochloa colona in Central America. Pesticide Science 51:341-346.

SMITH, R. J. JR. 1961. 3,4-Dichloropropionanilide for control of barnyardgrass in rice. Weeds 3:318-322.

TOSQUY, O. H.; AYÓN, E. A.; VARGAS, A. B.; ESQUEDA, V. A.; VÁSQUEZ, A. 2002. Control de malezas y fertilización química y biológica en variedades de arroz. In: SOMEFI (ed.). Memoria del XIX Congreso Nacional de Fitogenética. Saltillo, Coah. México. p. 414.

VALENT. 2000. Regiment (bispyribac-sodium) a new postemergence rice herbicide. Technical Information Bulletin. Valent USA Corp. 4 p. 